\section{Power laws for the perception of rotation and the oculogyral illusion*}

\author{
WILLIAM ELSNER $\dagger$ \\ San Jose State College, San Jose, California 95114
}

Magnitude estimation was used to measure subjective motion for two indicators of vestibular function. Twelve Os made estimates of 5 -sec pulses of angular acceleration across the range of angular acceleration $X$ time (at) $=10-150 \mathrm{deg} / \mathrm{sec}$. Results were: (1) the power law describes subjective motion for all individual Os, (2) the power function exponent (1.41) for the perception of rotation is slightly greater than the exponent (1.25) for the oculogyral illusion, (3) a significant number of Os gave higher exponents for the perception of rotation, and (4) the magnitude estimates of the oculogyral illusion and perception of rotation were highly correlated within and across Os.

The purpose was to describe and compare the subjective sensation resulting from angular acceleration (a) in degrees/second ${ }^{2}$ by means of two indicators of vestibular function, the perception of rotation in the dark (POR) and the oculogyral illusion (OGI). Although psychophysical functions seem not to have been determined for the OGI, the POR has been described by a psychophysical equation in three studies. Brown (1966), using the procedure of magnitude estimation (Stevens, 1957), found that Stevens's power law described the first effect of prolonged constant a in the dark. Brown (1968) later confirmed those results with a cross-modal matching procedure. Clark and Stewart (1968a), using different values of prolonged constant a, found results that led them to question whether Stevens's power law applies to prolonged constant a. Clark and Stewart's (1968a) hypothesis concerning prolonged constant a may well be true, but their method of stimulus presentation introduces various constraints and response biases. In the present experiment relatively short 5 -sec pulses of a were used as stimuli to be evaluated by magnitude estimation. Short pulses are not subject to many of the variables that affect prolonged constant a.

\section{Apparatus}

\section{METHOD}

Os were rotated in the Ames man-carrying rotation device (MCRD). The MCRD is a simulator with one degree of freedom which rotates about a vertical axis

*This study was conducted at NASA's Ames Research Center, Moffett Field, California under National Aeronautics and Space Administration Grant NGL 05-046-002 to San Jose State College, Brant Clark, principal investigator, and John Stewart, technical monitor.

†Requests for reprints should be sent to William Elsner, Box 511 , University of Florida College of Medicine, Gainesville, Florida 32601. with angular acceleration increments to $30 \mathrm{deg} / \mathrm{sec}^{2}$ for a range of durations limited only by the maximum speed of $45 \mathrm{rpm}$. The accelerations are monitored by ink writing recorders driven by onboard angular accelerometers. The cockpit rotates on the shaft of a dc motor which is controlled by voltage signals from an analog computer, making it possible to vary accelerations in a small fraction of a second. The rotation is free from significant vibrations, particularly at the lower angular velocities $( \pm 20 \mathrm{rpm})$ used. $O$ sits at the center of rotation in a special seat within a lightproof cockpit, $5 \mathrm{ft}$ wide, $7 \mathrm{ft}$ long, and $5.5 \mathrm{ft}$ high. The room is ventilated by two fans, which also serve to mask external noises. An aviator's helmet, shoulder harness, and lap belt are used as safety precautions (Clark \& Stewart, 1968a).

The visual target for the OGI was a cubical frame of wire measuring $10 \mathrm{~cm}$ on each side; it was mounted at $O$ 's approximate eye level and $3 \mathrm{ft}$ in front of him. The cube was coated with luminescent paint and was illuminated by ultraviolet light. The wire sections which formed the back part of the cube were painted black, leaving only nine edges visible. This complex form reduces autokinesis (Graybiel \& Hupp, 1946).

\section{Observers}

Os were 12 men, between 21 and 40 years of age, who were found to be in good health and to show normal responses to caloric stimulation of the ear. All Os had previously participated in one study in which cupulograms and/or absolute thresholds were determined. No Os had previously participated in a study of magnitude estimation.

\section{Procedure}

The Os were instructed concerning magnitude estimation and the concept of a ratio. They were then given practice with model magnitude estimation scales. The Os were also told that after the first stimulus presentation in the MCRD they were to choose a modulus (an arbitrary numerical value given to a standard) which would make ratio judgments as easy as possible (Engen \& Ross, 1966), but they were restricted to numbers between 10 and 100 for their modulus. They were told that decimals were permitted but a magnitude estimate of zero was not allowed, nor were the Os asked to denote the direction of rotation. No blank trials were included.

$O$ sat erect in the seat of the MCRD throughout all trials, with his helmet pressed against a U-shaped headrest which maintained his head in an erect fixed position. For the POR condition the $O$ was in complete darkness and attended to the magnitude of his subjective sensation of rotation; for the OGI condition the 0 attended to the magnitude of the perceived motion of the illuminated cube. Following a ready signal, the $O$ was given a 5 -sec pulse of angular acceleration. Since it is well known that in the vestibular system subjective sensation increases over time during and after a constant angular acceleration (Brown, 1966; Clark \& Stewart, 1968a; Parsons, 1968), the O's task was to report the magnitude of subjective sensation within $6 \mathrm{sec}$ after the end of the pulse, as signaled by $E$ using the word "reply."

The standard angular acceleration $X$ time $(\mathrm{at})=60 \mathrm{deg} / \mathrm{sec}$ was a stimulus easy to remember and was in the approximate geometric center of the stimulus range (cf. Poulton, 1968). The O's standard was presented twice before each experimental session, once in each direction, and it was constant for all Os. The $O$ was informed that the first two stimuli of each experimental session constituted his standard.

The nine stimuli used were: at $=10,15$, $25,40,60,75,90,120$, and $150 \mathrm{deg} / \mathrm{sec}$, and the intertrial intervals that followed each stimulus presentation were: $1,1,2,2$, $2,2 \frac{1}{2}, 3,3$, and $3 \mathrm{~min}$, respectively. The intertrial intervals following the beginning of a stimulus presentation were determined from the adaptation decay curves of Parsons (1968) and designed to permit the effects of one stimulus to dissipate before another was presented. The stimuli were varied randomly within a block of nine trials, and the direction of angular acceleration was varied systematically to maintain the MCRD velocity within $\pm 20 \mathrm{rpm}$. The trials for each experimental condition (OGI and POR) were presented to the $O$ during 25 -min sessions with 25 min between sessions. The two standard stimuli and one block of trials were 


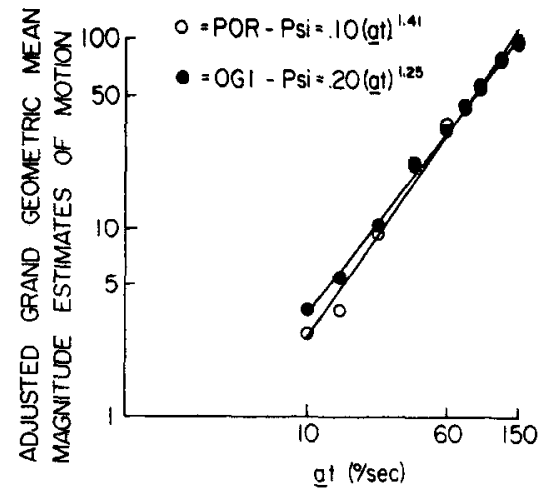

Fig. 1. Lines of best fit determined by least squares for the geometric mean magnitude estimates of motion as a function of at $(\mathbf{N}=12)$.

presented to the $O$ in each session, making a total of 11 trials per session. The first block of trials was a practice block in order to familiarize the 0 with the procedure and eliminate the largest part of habituation. For each experimental condition the total number of blocks was six per $\mathrm{O}$; therefore, each stimulus, including a stimulus equal to the standard, was estimated six times. The last five blocks only were used in the analysis of the results. Six Os judged the POR first, the other six Os judged the OGI first. Each $O$ finished a complete experimental condition before beginning his second experimental condition.

\section{RESULTS}

Averaging Within and Across $\mathrm{Os}$

Since each $O$ in the present study was free to choose the modulus that best matched his perception of the standard, there was attendant variation in the choice of moduli. Consequently, the data for each 0 were averaged according to a method devised by Lane, Catania, and Stevens (1961). First, all the magnitude estimates from the experimental trials were converted to logs and averaged to obtain a grand mean. Then the mean of the 45 estimates for each $O$ was subtracted from the grand mean of logs over the 120 s. The resulting difference was then added to the $\log$ of each of the Os' magnitude estimates. This method of averaging (Adair, Stevens, \& Marks, 1968) minimizes the influence of shifts in the subjective magnitude of the standard.

\section{Results Averaged Across All Os}

In Fig. 1 the geometric means for 12 Os are plotted as a function of at. The use of at for the stimulus dimension is merely a vestibular system formality, because multiplying by the constant duration of stimulus displaces the function on the abscissa of $\log$ - $\log$ coordinates and does not affect the slope of the function. The relationship between stimulus and
Table 1

Computed Constants of the Individual Os' Power Functions

\begin{tabular}{cccccc}
\hline & \multicolumn{2}{c}{ Perception of Rotation } & & \multicolumn{2}{c}{ Oculogyral lllusion } \\
\cline { 2 - 3 } Observer & Exponent & $\begin{array}{c}\text { Pearson } \\
\text { Coefficient of } \\
\text { Correlation }\end{array}$ & & Exponent & $\begin{array}{c}\text { Pearson } \\
\text { Coefficient of } \\
\text { Correlation }\end{array}$ \\
\hline 1 & 1.49 & .995 & 1.42 & .997 \\
2 & 1.01 & .996 & .97 & .994 \\
3 & 1.55 & .977 & 1.52 & .994 \\
4 & 1.91 & .988 & 1.59 & .991 \\
5 & 1.07 & .997 & 1.00 & .994 \\
6 & 1.76 & .984 & 1.34 & .995 \\
7 & 1.48 & .979 & 1.82 & .978 \\
8 & 1.19 & .976 & .03 & .962 \\
9 & 1.46 & .974 & .90 & .981 \\
10 & 1.67 & .980 & 1.18 & .990 \\
11 & 1.07 & .991 & 1.01 & .994 \\
12 & 1.22 & .989 & 1.22 & .990 \\
\hline
\end{tabular}

sensation for both the POR and the OGI is described by a straight line in the log-log coordinates. The Pearson $r$ between the log stimulus and $\log$ magnitude estimate for the POR was .995, and for the OGI was 999. This confirms the hypothesis that the subjective sensation of motion for both the $P O R$ and the $O G l$ is described accurately by Stevens's power law, when pulses of a are used as stimuli.

The power function describing the relationship between sensation and stimulus, as determined by least squares, for the POR has the exponent 1.41, and for the OGI the exponent is 1.25 . It is clear that the POR and the OGI are highly correlated vestibular indicators in terms of the quantitative growth of sensation.

\section{Results for Individual Os}

Table 1 and Fig. 2, which contain the results for individual $\mathrm{Os}$, indicate that the increase of subjective vestibular sensation for individual Os conforms closely to Stevens's power law. The Pearson rs for 13 of the 24 functions were over .99 , and only one Pearson $r$ was below 97 . Although the growth of vestibular sensation with stimulus magnitude for ir tividual Os conforms to the power law, for the POR the range of exponents was 1.01-1.92, and for the OGI the range of exponents was .90-1.82.

Although the power function exponents for the POR and the OGI are similar and the magnitude estimates for the POR and the OGl correlate highly, the evidence that the POR and the OGl are almost identical indicators of vestibular sensation in their quantitative aspects was examined further. The power function exponents of both experimental conditions for all 120 s were compared by means of the Wilcoxon matched-pairs signed-ranks test for related

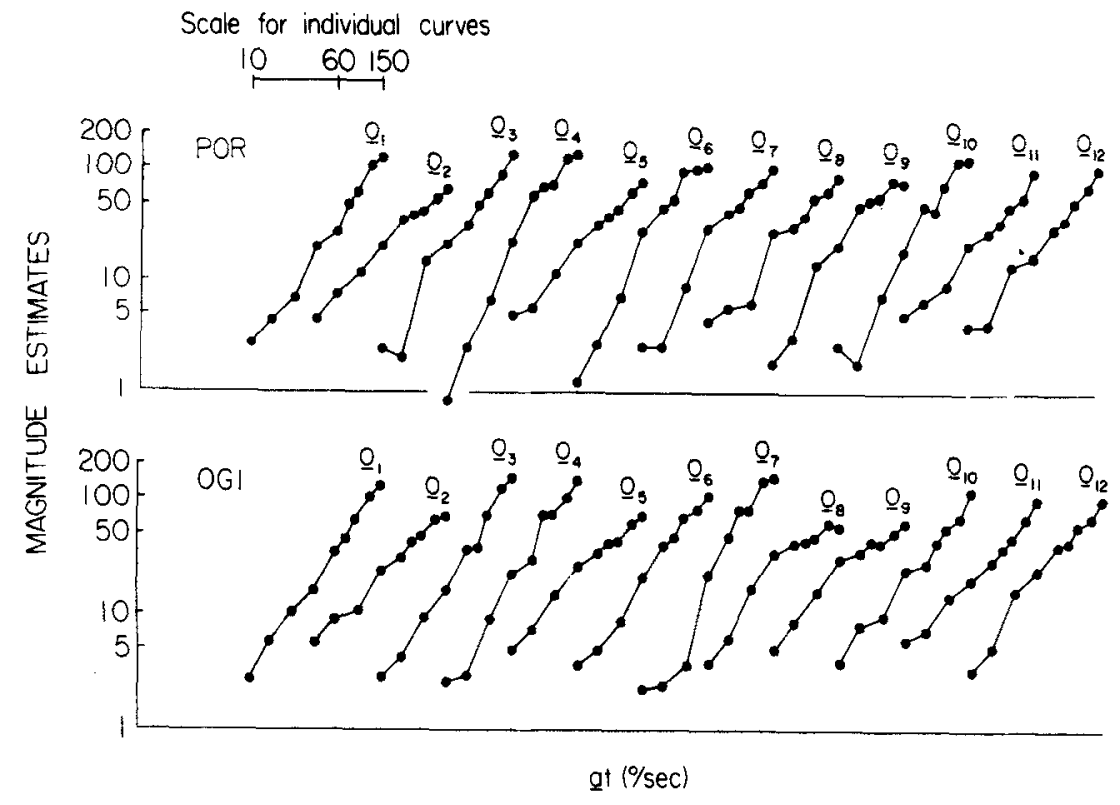

Fig. 2. Geometric mean magnitude estimates of the POR and the OGI for individual Os as a function of the nine at values. 
samples, and the difference was found to be significant $(T=10, p<.02$, two-tailed). There was a significant number of Os for whom the POR gave a higher exponent than the OGI.

The Spearman rank-order correlation coefficient (rho) was determined, using the power function exponents of the 12 Os for both the POR and the OGI. It was found to be significant (rho $=.70, p<.05$, one-tailed), indicating that an $\mathrm{O}$ having a relatively high POR power function exponent tends to have a high power function exponent for the OGI.

Since there is some evidence that previous experience with magnitude estimation influences subsequent experience (cf. Poulton, 1968), the Mann-Whitney $U$ test was also performed on the power function exponents of both the POR and the OGI experimental conditions separately. The power function exponents from each experimental condition were divided into two separate subsamples: (1) the six exponents that were determined before experiencing the second experimental condition, and (2) the six exponents that were determined after experiencing the first experimental condition. Neither the subsamples of exponents in the POR experimental condition $(U=18, p=.531)$ nor the subsamples of exponents in the OGI experimental condition $(U=14, p=.294)$ was significantly different. There is insufficient evidence for stating that previous experience with magnitude estimation contributed to the significant correlations within and across Os for the power function exponents of the POR and the OGI.

\section{DISCUSSION}

The power function exponent found in this study for POR of 1.41 differs from the 1.0 exponent found by Brown (1966, 1968). In addition, the conformity to the power law found in this study disagrees with the results of Clark and Stewart (1968a). There were several procedural differences between the present study and those previous studies that may account for the differences in results. The constant angular accelerations used as stimuli by Brown $(1966,1968)$ and Clark and Stewart (1968a) started at at $=0$ and extended over a prolonged time. These stimuli could have led to adaptation of the vestibular system (cf. Parsons, 1970), hysteresis effects (Stevens, 1957), or an ordinal scale instead of a ratio scale. In addition, a different stimulus range was used by Brown (1966, 1968) and Clark and Stewart (1968a), and it is well known that the power function exponent tends to be influenced by the range of stimuli when more than one estimate is made for each stimulus (cf. Poulton, 1968). In the present study a wide range of stimuli was used. Since the method of magnitude estimation underestimates the exponent because of the regression effect (cf. Stevens \& Greenbaum, 1966), the absolute value for the exponents of the POR and the OGI may be closer to 1.5 .

It is clear from Fig. 1 that the fit of the power function would not be greatly improved by the subtraction of a threshold constant from the stimulus or response dimension (cf. Marks \& Stevens, 1968). That procedure was used in Brown's studies $(1966,1968)$. In the present experiment the stimuli were sufficiently suprathreshold (cf. Clark \& Stewart, 1968b, c, 1969; Doty, 1969) so that the increased slope sometimes found at the low end of the stimulus range was not particularly apparent.

As the results of both Clark and Stewart (1969) and the present study suggest, the POR and the $O G l$ are relatively independent but overlapping (correlated) psychophysiological mechanisms. The crossing of the two functions at at $=75 \mathrm{deg} / \mathrm{sec}$ in Fig. 1 should not be interpreted as the point of equal sensation magnitude. The point of crossover is purely an artifact of the method of averaging and of the fact that the Os were required to use the same modulus for each experimental condition.

In conclusion, the results of this study warrant the statement that Stevens's power law provides an excellent description of the relationship of subjective vestibular sensation to stimulus pulses of angular acceleration. In addition, the subjective changes in sensation for the OGI and the POR at suprathreshold levels are similarly mediated psychophysiological processes with only a small degree of divergence.

\section{REFERENCES}

ADAIR, E. R., STEVENS, J. C., \& MARKS, L. E. Thermally induced pain, the dol scale, and the psychophysical law. American Journal of Psychology, 1968, 81, 147-164.

BROWN, J. H. Magnitude estimation of angular velocity during passive rotation. Journal of Experimental Psychology, 1966, 72, 169-172.

BROWN, J, H. Cross-modal estimation of angular velocity. Perception \& Psychophysics, 1968, 3, 115-117.

CLARK, B. Thresholds for the perception of angular acceleration in man. Aerospace Medicine, 1967, 38, 443-450.

CLARK, B., \& STEWART, J. D. Magnitude estiniates of rotational velocity during and following prolonged increasing, constant, and zero acceleration. Journal of Experimental Psycholegy, 1968a, 78, 329-339.

CLARK, B., \& STEWART, J. D. Comparison of three methods to determine thresholds for the perception of passive, bodily angular acceleration. American Journal of Psychology, 1968b, 81, 207-216.

CLARK, B., \& STEWART, J. D. Comparison of sensitivity for the perception of bodily rotation and the oculogyral illusion. Perception \& Psychophysics, 1968c, 3, 253-256.

CLARK, B., \& STEWART, J. D. Effects of angular acceleration on man: Thresholds for the perception of rotation and the oculogyral illusion. Aerospace Medicine, 1969, 40, 952-956.

DOTY, R. L. Effect of duration of stimulus presentation upon the angular acceleration threshold. Journal of Experimental Psychology, 1969, 80, 317-321.

ENGEN, T., \& ROSS, B. M. Effect of reference number on magnitude estimation. Perception \& Psychophysics, 1966, 1, 74-76.

GRAYBIEL, A., \& HUPP, D. I. The oculogyral illusion. Journal of Aviation Medicine, 1946, 17, 3-27.

LANE, H. L., CATANIA, A. C., \& STEVENS, S. S. Voice level: Autophonic scale, perceived loudness, and effects of side tone. Journal of the Acoustical Society of America, 1961, 33 , 160-167.

MARKS, L. E., \& STEVENS, J. C. The form of the psychophysical function near threshold. Perception \& Psychophysics, 1968, 4, 315-318.

PARSONS, R. D. Magnitude estimates of the oculogytal illusion during and following angular acceleration. Journal of Experimental Psychology, 1970, 84, 230-238.

POULTON, E. C. The new psychophysics: Six models for magnitude estimation. Psychological Bulletin, 1968, 69, 1-19.

STEVENS, S. S. On the psychophysical law. Psychological Review, 1957, 64, 153-181.

STEVENS, S. S., \& GREENBAUM, H. B. Regression effect in psychophysical judgment. Perception \& Psychophysics, 1966, 1 . 439-446.

(Accepted for publication August 31, 1970.) 